\title{
Warehouse Security System based on Embedded System
}

\author{
Gen Li \\ Department of Electronic Engineering, Tianjin University \\ of Technology and Education \\ Tianjin 300222, China \\ Email:996771037@qq.com \\ Jianmin Zhang \\ Department of Electronic Engineering, Tianjin University \\ of Technology and Education \\ Tianjin 300222, China \\ Email: bjmzhang628@eyou.com
}

\begin{abstract}
This paper designs a security system for warehouse's security. Based on linux and S3C2440, this system's peripheral devices include temperature sensors, humidity sensors, cameras, web clients, Android clients, fans, and led. It uses the temperature sensor to monitor the temperature and fire, uses camera to real-time monitor whether there are unsafe factors. If the system detects that the room temperature is too high or too low, it will automatically alarm the security staff and automatically open the fan. Through the camera real-time monitoring, the security staff might be suspicious persons or has unexpected events in the warehouse. The video surveillance also will capture real-time video data and transfer data to Android client, then security personnel will control the security information whenever and wherever if they need. If the warehouse breaks out of fire, thief, accident, and so on, this system will take automatic protective measures, capture video evidence, and warn the security staff. Finally, if the warehouse has an accident, the system will reduce the damage to the lowest.
\end{abstract}

Keywords-Security system; Linux; Embedded; Remote control; Android

\section{INTRODUCTION}

After the system start, first, initialize the mutex lock and other global variables, and then open 4 threads: camera, serial port and Android.

Camera thread drives camera module to initialize equipment, then captures the image and places it in the shared memory. Then it will judge the client if it has a real-time monitoring command, if there is, it sends some words to the client through the network and stores the picture to the database. Otherwise, it stores the pictures to the database and then puts the picture in the shared

\author{
Minghua Niu \\ Department of Electronic Engineering, Tianjin University \\ of Technology and Education \\ Tianjin 300222, China \\ Email:981859678@qq.com \\ Yong Li \\ Sichuan University of Science \& Engineering \\ Sichuan 643000, China \\ Email: 2603127446@qq.com
}

memory; Serial thread initialize the M0 module, sets the baud rate as 115200,8 data bits, 0 parity bit and 0 stop bit. It reads the values of temperature sensor and the humidity sensor. Afterwards, determine whether the temperature and humidity is normal. If there is some Abnormal, it will sends a alarm message to the user mobile phone remotely through internet; Android thread drives Android module to initialize server and monitors messages. It will judge if there has a new connection or a new command, if there is, it will establish a TCP link to receive commands and transfers datas. Otherwise, it will still monitor systemall time.

\section{THE DESIGN OF SYSTEM NODULES'}

\section{FUNCTION}

\section{A. Camera module}

This module is used to control the camera to read the image frame and stores the read-data into the shared memory. The main process function gets the data from the shared memory to process for further steps. In this system, the camera will monitor screen in real-timely.

In Linux, the video device is the device file, and it can be accessed like ord inary files such as reading and writing, the camera in the folder of /dev/video1. Video4Linux2 $(\mathrm{V} 4 \mathrm{~L} 2)^{[1-3]}$ is an application programming interface for audio and video image. This module uses Video4Linu x2's application functions and data organization to acquire the USB camera image under the Linux system.

Firstly, the system opens the camera equipment according to the name or number of devices. Then it initializes the video equipment and sets picture format size, frame format, the camera capture mode and distributes cache. Later, it realizes the application and mapping of the Image frame buffer. Finally, the camera module captures the image and transforms YUYV format to RGB24 format, then transforms RGB24 format to BMP format. At last, it 


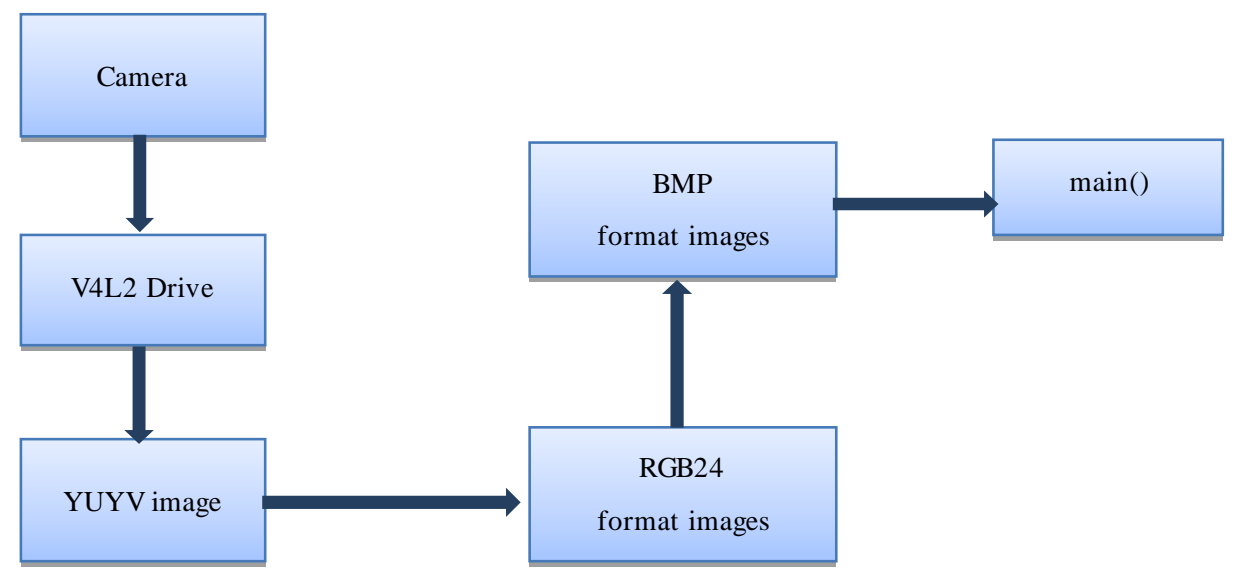

Figure 1. Camera flow chart

The notes of V4L2 :

This module uses V4L2 technology and USB interface; it needs to configure the kernel driver. Configuration is shown below:

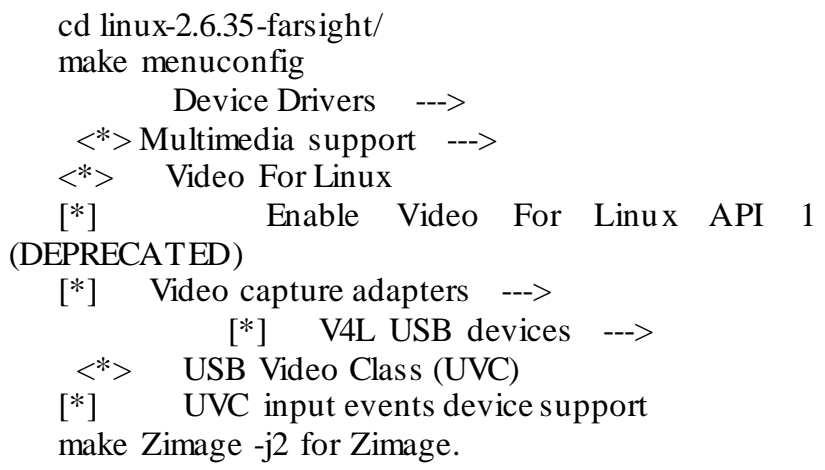

The V4L2 specific procedures:

1. Open the equipment by the block way.

2. Obtain equipment capability, look at equipment s' function.

3. Set the standard and the frame format, including PAL, NTSC. Frame format's width and height.

4. Application cache for devices.

5. Transform the frame buffer to physical address, and mapping to user space, so that it can be operated directly collected frame, without having to copy.
6. Apply all frame buffers into the queue, so that store the collected data.

7. Start the collection of video.

8. Remove frame buffer data which have been collected from the queue, acquire the original collection data.

9. Move the buffer to the end of queue again, so you can loop acquisition.

10. Stop the video collection.

11. Close the video equipment.

\section{B. The GPRS module}

When the S3C2440 receives the warning information, firstly, open the serial port equipment, set the baud rate serial port initialization, data bits, parity bit and stop bit, and then sends the control commands and information to the GPRS module, and finally closes the serial port. GPRS will send the alarm information to the mobile phone.

\section{MO control module}

The M0 module acquires the temperature and humidity information through a temperature sensor and humidity sensor, and then changes the data with data through the serial port. Furthermore, the M0 module can receive the control command from Android and PC, Stores and sends the temperature and humidity data to the network. So the system can control the led and fan in the warehouse through the data in the network. The detailed steps are shown in Fig. 2. 


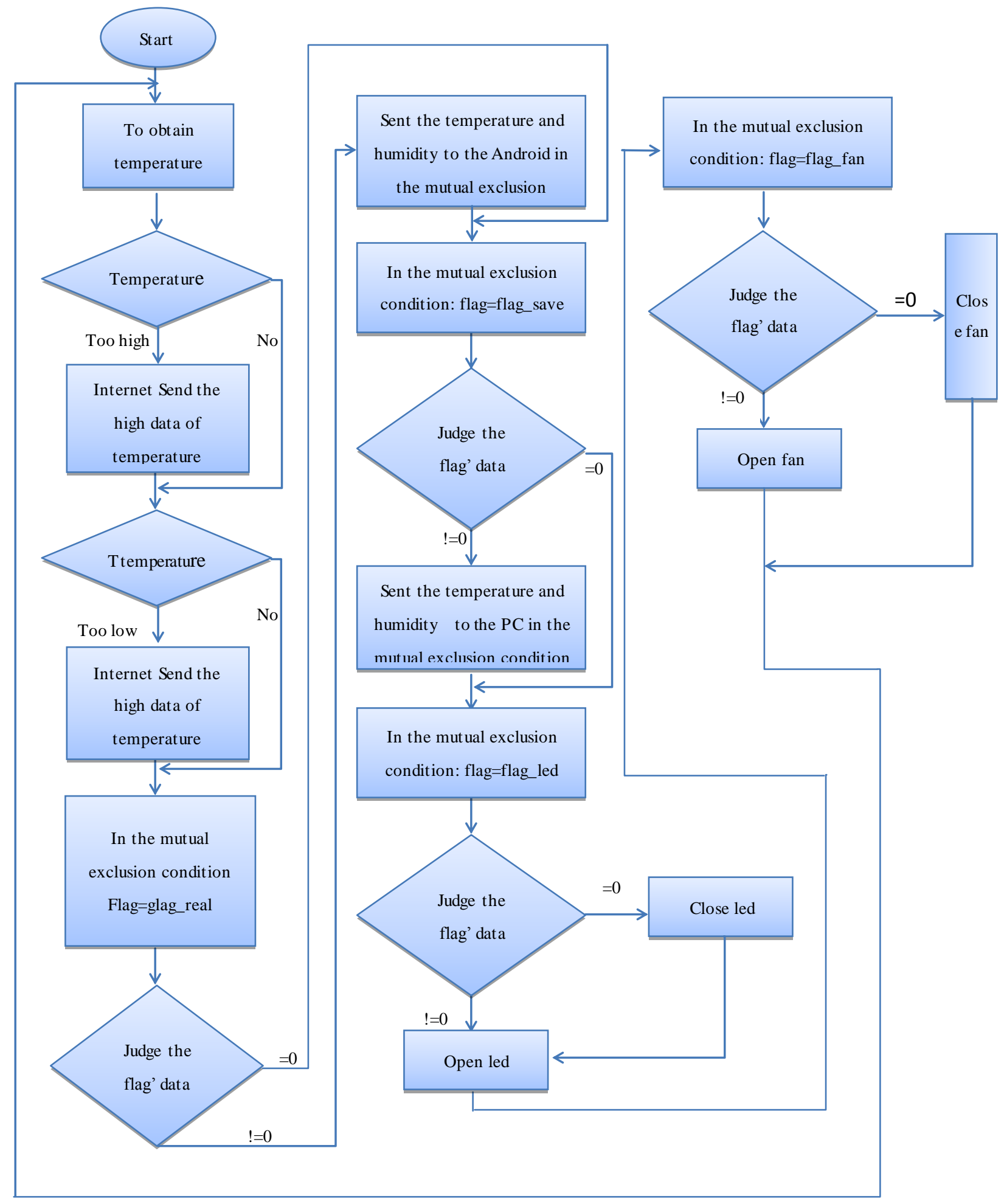

Figure 2. Flow chart of MO control 


\section{The network module}

This module is used for sending video and temperature and humidity to the database, Android client and web client. The Android client and web client also can sent command to this module to control the fan and $\mathrm{LED}^{[7]}$.

The detailed steps are shown in Fig. 3.

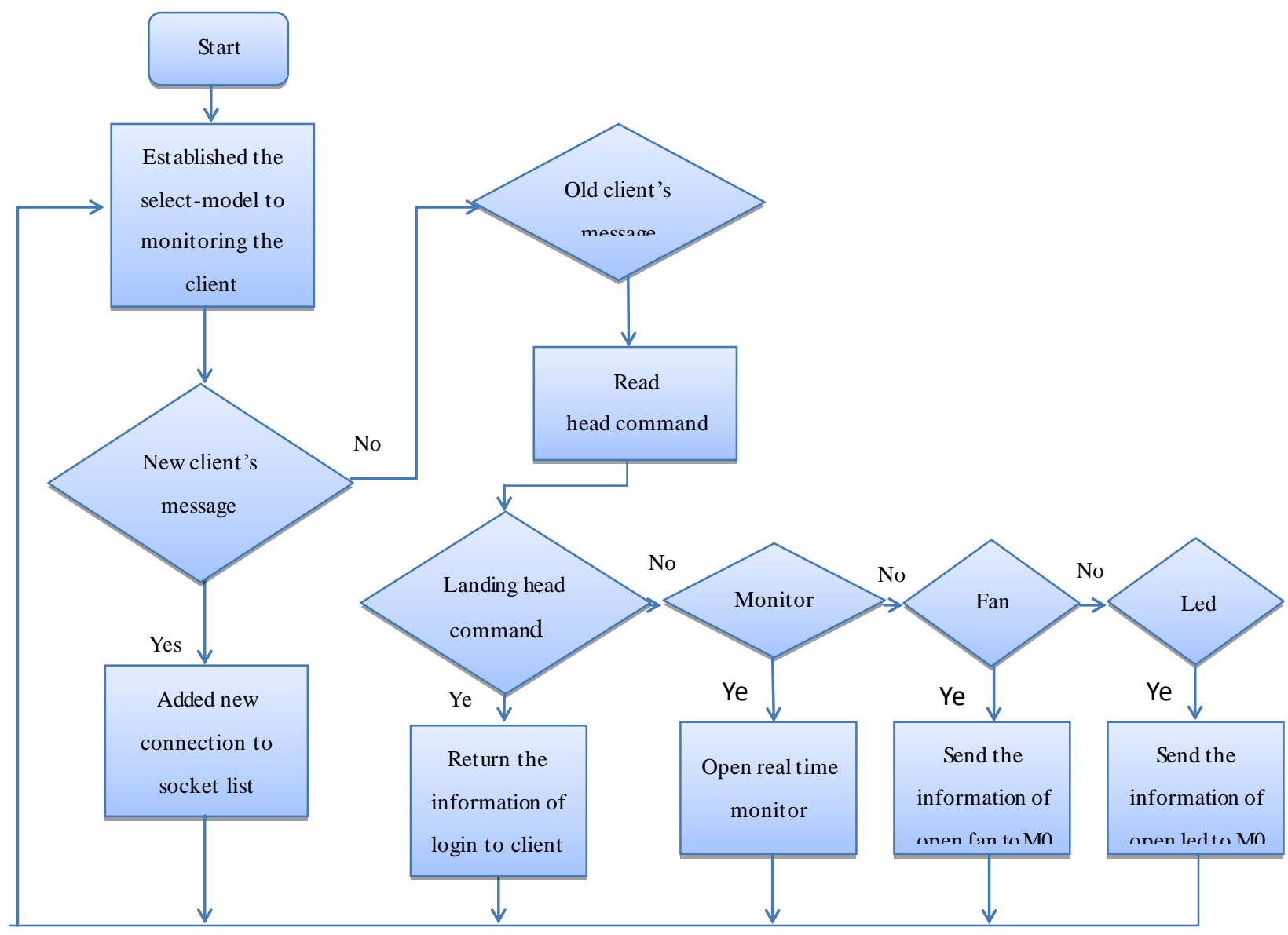

Figure 3. Flow chart of net work module 
In above figure, after network module operates, first establish the select-model to monitor the client. If this is a new client link, it will be added new connection to socket list and returns the select model; if it is an old user links, check whether the client sends a message, if sends and this module received information is read command head. To determine whether the login command, if it is the landing and will return the information to the select model, or to judge whether the real-time monitoring; if it is a real-time monitoring of open real-time monitoring signs and returns the select model, or to determine whether the fan control command; if it is fan control command, send control commands to the M0 fan and return to select model otherwise, to determine whether the control command is led, then sends the control command to the M0 to return led select model, otherwise it returns select model.

\section{E. Web server module}

This module is used for Web Webpage responses from client requests, including $\mathrm{Web}$ page login and exit function, registration or delete user or modify password, Web real-time monitoring, check the Web monitoring recording, control the switch of LED lights and fan from Web page ${ }^{[8][9]}$.

The web server work flow: first initialize the data area and the server, when a new request or order the command parsing, this instruction has five cases: A. Request landing. B. Login error. C. Send a control command to the server. D. Call the database to obtain data interface (read the image, temperature, humidity etc.). E, Store the interface by call the database (save picture, temperature, humidity etc.). If there is login error, then are logins. If the login is successful, to determine whether the root user, if it is, then add, delete users to modify the server, other users password etc. If you're not a root user, just ordinary users, you can only modify the corresponding table and character segment the user pass word and update the database $\mathrm{e}^{[10]}$.

\section{F. The Android client}

This module is used to display the temperature, humidity data, control the monitoring, control the video, fan and led equipment, send and receive with the server. It includes four functions: MainAct, MoinAct, ClientAct and $\operatorname{RegAct}^{[10-13]}$

The main interface (MainAct): When you open the Android client, firstly, it appears landing interface, then initialize get each view object instance, connect to the server to get the socket object, initialize the MyApplication get handler, then open thread, circulation receives from the server information. Detection of landing button has pressed, if it has, sent to the server login information, if not, to detect whether the received handler login information is correct, incorrect information will continue to wait, if it is correct, the jump to the MoniActivity.

The monitoring interface (MoinAct): After start equipment, initialize each view instance, get a handler object, get ready to accept the message, and refresh the image View picture if message is the picture, if message is the temperature, humidity, temperature, humidity display information.

Customer interface (ClientAct): First, read the 10 head bytes of Command, then detect whether the login command, if it is, sending message to mainActicity, else detecting whether the login command head, if it is to send message to MoniActivity, or to detect whether the image is of the head of the order, if it is to send message to MoniActivity, detection of temperature, humidity or whether it is the head of the order, then sends a message to the moinactivity.

Registration interface (RegAct): First, initialize each view, get instantiated object, receive input account and pass word, then the two test input password is consistent, if consistent, send the account number and password to the server, or to re input. If the return is the end of the correct server login registration process, or continue to enter the account pass word.

\section{CONCLUSIONS}

This paper mainly completes a warehouse security system based on Linux System. It contains temperature sensor, humidity sensor, surveillance module, M0 control module, the web server, the master server, the web client, and Android client. This system can help enterprise to administrate warehouse, make sure the safe of it by a variety of measures, and can real-time operation remotely. It can automatically alarm the danger in time and control the video surveillance to send real-time video data to a web server or Android client used by security personnel. They can monitor and control warehouse related facilities in order to protect the safety of the warehouse to the maximum extent

\section{REFERENCE}

[1] Michael H Schimek,Bill Dirks,Hans Verkuli. Video for Linuxtwo API specification http://v412spec.bytesex.org/v412spec/v412.pdf . 2008

[2] Dirks B.Video for Linux Two API Specification.

[3] Samsung Electronics.S3C2410A 200MHz\&266MHz 32-bit RISC Microprocessor USER'SMANUAL Revision 1.0. . 2004

[4] Dirks B.Video for Linux T wo API Specification.

[5] Video4Linux Kernel API Reference. http://inux.bytesex.org/v412/API.html

[6] WANG Fei,KONG Cong Realization of Linux camera driver based on V4L2[J]. Electronic Science and technology. 2012(02)

[7] Hinton G E,Salakhut dinov R R.Reducing the dimensionality of dat a with neural networks[J].Science,2006(5786):504-507

[8] ]XU Hong-hua,LIU Ke. Development and application of controller based on embedded Sever Web Industrial computer 2004/04

[9] Guan Mo;Gu Minghai Design and implementation of an embedded web server based on ARM 20105

[10] Hammel M J Mongoose:an embeddable Web server 2010(192)

[11] ISO base media file format[S/OL].ISO/IEC14496-12. 2008

[12] Shiddiqi A M,Pratama H,Ciptaningtyas H T.A Video StreamingApplication Using Mobile Media Application Programming Interface. Telkomnika . 2010

[13] Gavalas D,Economou D.Development Platforms for Mobile Ap-plications:Status and Trends. IEEE Software . 2011 